\title{
铜/凹凸棒石复合材料高效吸附放射性磑离子性能
}

$$
\begin{array}{r}
\text { 余祥坤 }^{1} \text {, 刘 坤 }{ }^{1} \text {, 李志鹏 }{ }^{1} \text {, 赵雨露 }{ }^{1} \text {, 沈锦优 }{ }^{2}, \\
\text { 茆 平 }{ }^{1} \text {, 孙爱武 }{ }^{1} \text {, 蒋金龙 }{ }^{1}
\end{array}
$$

(1. 淮阴工学院化学工程学院, 矿盐资源深度利用技术国家地方联合工程研究中心, 江苏省凹土资源利用重点实 验室, 淮安 223003; 2. 南京理工大学 环境与生物工程学院, 江苏省化工污染控制与资源化高校重点实验室, 南京 210094)

摘 要: 放射性碘是核裂变必然产物。为解决现有铜基吸附剂的吸附容量低、铜利用率低、易脱附等不足, 基于凹 凸棒石的廉价易得、可交换阳离子富足和层间结构等特性，采用浸渍-还原法将单质 $\mathrm{Cu}$ 负载于凹凸棒石结构中，制 备得到 $\mathrm{Cu}$ /凹凸棒石复合材料, 对样品进行表征并考察样品对液相碘离子的吸附性能。研究结果表明, 实验制备的 $\mathrm{Cu}$ /凹凸棒石复合材料约有 $7.5 \%$ 掺杂量的纳米铜颗粒负载于凹凸棒石层间结构中。与传统铜基吸附剂相比, 该材料 具有高达 $116.1 \mathrm{mg} / \mathrm{g}$ 的碘离子吸附量以及 $72 \%$ 铜利用率; 并且可在中性、弱酸性以及 $\mathrm{SO}_{4}{ }^{2-} 、 \mathrm{NO}_{3}{ }^{-} 、 \mathrm{Na}^{+} 、 \mathrm{Mg}^{+}$等 干扰离子共存的环境下使用。此外, 得益于铜负载凹凸棒石结构内, 该复合材料展现出较强的抗氧化性能, 且吸附 后产物也具有较强的稳定性。

关 键 词：凹凸棒石; 铜; 放射性碘离子; 脱附率; 吸附容量

中图分类号: TB333 文献标识码: A

\section{Efficient Adsorption of Radioactive Iodide by Copper/Palygorskite Composite}

\author{
YU Xiangkun ${ }^{1}$, LIU Kun ${ }^{1}$, LI Zhipeng ${ }^{1}$, ZHAO Yulu ${ }^{1}$, SHEN Jinyou ${ }^{2}$, \\ MAO Ping ${ }^{1}$, SUN Aiwu ${ }^{1}$, JIANG Jinlong ${ }^{1}$
}

(1. Key Laboratory for Palygorskite Science and Applied Technology of Jiangsu Province, National \& Local Joint Engineering Research Center for Mineral Salt Deep Utilization, School of Chemical Engineering, Huaiyin Institute of Technology, Huaian 223003, China; 2 Jiangsu Key Laboratory of Chemical Pollution Control and Resources Reuse, School of Environmental and Biological Engineering, Nanjing University of Science and Technology, Nanjing 210094, China)

\begin{abstract}
To solve the disadvantages of low adsorption capacity, low utilization and easily desorption of traditional $\mathrm{Cu}$-based adsorbents for radioactive iodide, the inevitable product of nuclear fission, $\mathrm{Cu} / \mathrm{Palygorskite}$ composite $(\mathrm{Cu} @ \mathrm{PAL})$ was synthesized through impregnation-reduction method, characterized and applied to remove $\mathrm{I}^{-}$anions
\end{abstract}

收稿日期：2020-11-19; 收到修改稿日期：2021-01-04; 网络出版日期：2021-03-01

基金项目：国家自然科学基金(51908240); 江苏省自然科学基金(BK20181064); 江苏省六大人才高峰资助项目(2018-JNHB-009); 江苏省高校自然科学基金重大项目(18KJA430006); 江苏省研究生科研与创新计划项目(SJCX20_1331); 江苏省 凹土资源利用重点实验室开放课题(HPK201905)；2020 年省级(202011049042Y)、校级(202011049011XJ)大学生 创新创业训练计划项目

National Natural Science Foundation of China (51908240); Natural Science Foundation of Jiangsu Province (BK20181064); Six Talent Peaks Project in Jiangsu Province (2018-JNHB-009); Natural Science Key Project of the Jiangsu Higher Education Institutions (18KJA430006); Postgraduate Research \& Practice Innovation Program of Jiangsu Province (SJCX20 1331); Foundation of Jiangsu Provincial Key Laboratory of Palygorskite Science and Applied Technology (HPK201905); Provincial (202011049042Y), University-level (202011049011XJ) Innovation and Entrepreneurship Training Program Project for Undergraduate

作者简介：余祥坤(1993-), 男，硕士研究生. E-mail: yxk0079@163.com YU Xiangkun(1993-), male, Master candidate. E-mail: yxk0079@163.com

通信作者: 芗 平，校聘副教授. E-mail: pingmao@hyit.edu.cn; 孙爱武，教授. E-mail: sunaiwu@hyit.edu.cn MAO Ping, associate professor. E-mail: pingmao@hyit.edu.cn; SUN Aiwu, professor. E-mail: sunaiwu@hyit.edu.cn 
based on the excellent traits of PAL, such as cheap, abundant exchangeable cations and interstratified structure. Results indicate that there are about $7.5 \mathrm{wt} \%$ copper nanoparticles loaded into PAL for the as-prepared composite. Cu@PAL has better performance in the adsorption of iodide as compared with traditional $\mathrm{Cu}$-based adsorbents, with $72 \%$ utilization efficiency and excellent adsorption capacity of $116.1 \mathrm{mg} / \mathrm{g}$. $\mathrm{Cu} @$ PAL is suitable for adsorption of $\mathrm{I}^{-}$anions under the conditions of neutral, weak acid and the coexistence of interference ions. In addition, the composite exhibit strong oxidation resistance, and the adsorbed product also has strong stability due to the copper stored in the PAL structure.

Key words: palygorskite; elemental copper; radioactive iodide; desorption efficiency; adsorption capacity

随着化石能源消耗引起的污染问题日益严峻, 核电发展越来越受重视 ${ }^{[1]}$ 。而核裂变必然产物, 放射 性碘产生的辐射会使生物机体受损和代谢紊乱从而 诱发癌症。碘离子是碘在水溶液中主要存在形式 ${ }^{[2]}$ 。 此外，放射性碘也常用于放射治疗、生物实验和医 学诊断等领域 ${ }^{[3]}$, 因而有效处理溶液中放射性碘离 子对于核电和放射核素的安全利用尤其重要。

处理放射性碘离子的传统方法有膜分离、离子 交换、吸附和生物处理等 ${ }^{[4-7]}$ 。膜分离是利用压力差 分离污染离子, 但膜材料易受污染 ${ }^{[8]}$ 。离子交换是通 过交换剂中阴离子与碘离子的交换实现碘离子固化, 但易引入其他污染离子 ${ }^{[9]}$ 。生物处理 ${ }^{[10]}$ 是利用微生 物或植物对碘离子的吸收富集碘离子，但较弱的吸 收性能以及辐射对生物体的破坏限制了该方法的实 际应用。吸附法 ${ }^{[11]}$ 是一种高效清洁、易于操作的方 法，适合于大批量废水的连续操作，其中利用活性 炭、沸石等吸附剂的物理吸附, 存在吸附效率低、 易脱附、选择性差等缺陷; 而利用金属或其化合物 与碘离子反应形成化学键来固化碘离子的化学吸 附 ${ }^{[12]}$, 恰好能弥补上述缺陷。已知的有铜基 ${ }^{[12]}$ 、银 基 ${ }^{[8]}$ 、铋基 ${ }^{[13]}$ 、铅基 ${ }^{[14]}$ 和录基 ${ }^{[15]}$ 等吸附材料。铅基、 采基吸附材料具有较高的重金属毒性和致癌性, 目 前已鲜有相关研究报道; 银基、铋基吸附材料成本 较高。因此, 储量丰富、价格低廉、毒性小的铜基 吸附剂是较为理想的碘离子吸附材料。但研究发现 只有亚铜对碘离子有吸附性能, 而表面的亚铜极易 被氧化生成惰性 $\mathrm{CuO}$ 膜 ${ }^{[12]}$, 从而限制了铜的利用率 和对碘离子的吸附性能。因此, 如何降低亚铜被氧 化几率、提升亚铜利用率是实现铜基吸附剂高效利 用的关键。

凹凸棒石 $(\mathrm{PAL})$ 是一种具有链层状结构的天然 富镁铝硅酸盐粘土矿物, 理论分子式为: $\left(\mathrm{Al}_{2} \mathrm{Mg}_{2}\right) \mathrm{Si}_{8} \mathrm{O}_{20}(\mathrm{OH})_{2}\left(\mathrm{OH}_{2}\right)_{4} \bullet 4 \mathrm{H}_{2} \mathrm{O}$ 。基本结构单位由 两层硅氧四面体夹一层镁/铝氧八面体构成, 其链层 间形成独特的一维孔道, 孔道四周分布有大量羟基
和氧原子, 孔道中存在的沸石水以氢键结合吸附水 分子。由于其拥有大量的可交换阳离子和发达的孔 隙结构且价格低廉, 常被用作吸附剂或载体 ${ }^{[16]}$ 。因 而, 本工作选用 PAL 为载体, 利用其结构中大容量 可交换阳离子对铜离子交换实现高效牢固负载, 同 时, 狭窄的孔道结构可降低铜被氧化速率和产物 $\mathrm{CuI}$ 的分解率, 价廉的 PAL 也可降低碘离子吸附剂 的成本。通过浸渍法将铜离子负载于 PAL 结构中, 再利用氢气还原制备出铜改性凹凸棒石复合材料 $(\mathrm{Cu} @ \mathrm{PAL})$, 研究了 $\mathrm{Cu} @ \mathrm{PAL}$ 对溶液中碘离子的吸 附性能。同时, 研究表征了吸附前后的复合材料, 分 析了 $\mathrm{Cu}$ 的赋存形态、负载机理以及对碘离子的吸 附机理。

\section{1 实验方法}

\section{1 实验试剂}

PAL 来源于江苏旰眙龙王山，使用前通过湿法 纯化处理。硝酸铜、碘化钠、盐酸、氢氧化钠、硝 酸钠、硫酸钠、碳酸钠和氯化钠均为分析纯, 来源 于国药集团化学试剂有限公司。

\section{$1.2 \mathrm{Cu} @$ PAL 的制备}

将 $50 \mathrm{~g}$ 凹凸棒石黏土矿浸泡于 $0.5 \mathrm{~L}$ 去离子水 中 $30 \mathrm{~min}$, 使用高速摚拌器以 $8000 \mathrm{r} / \mathrm{min}$ 搅拌 $20 \mathrm{~min}$, 制成凹凸棒石黏土浆料, 利用离心机将浆 料离心分离, 刮取细软色浅的上层黏土, 冷冻干燥, 研磨成粉，即为湿法纯化的凹凸棒石。

将 $5 \mathrm{~g}$ 上述纯化后的凹凸棒石样品超声分散于 $50 \mathrm{~mL} 0.2 \mathrm{~mol} / \mathrm{L} \mathrm{Cu}\left(\mathrm{NO}_{3}\right)_{2}$ 溶液中, 在 $60{ }^{\circ} \mathrm{C}$ 下搅拌 反应 $5 \mathrm{~h}$ 。离心, 利用 ICP-AES 分析上层清液中残余 的 $\mathrm{Cu}^{2+}$ 浓度, 洗涤固体并在 $100{ }^{\circ} \mathrm{C}$ 下烘干, 研磨成 粉备用, 标记为 $\mathrm{Cu}^{2+} @ \mathrm{PAL}$ 。

将 $\mathrm{Cu}^{2+} @ \mathrm{PAL}$ 置于气体管式炉中, 通入氢气并 在 $300{ }^{\circ} \mathrm{C}$ 下还原反应 $6 \mathrm{~h}$, 冷却至室温, 即得 $\mathrm{Cu} @ \mathrm{PAL}$ 复合材料。 


\section{3 表征}

利用 FEI Q250 场发射扫描电子显微镜(SEM)和 FEI Tecnai G2 透射电子显微镜(TEM)分析材料的微 观形貌; 利用 D8 Discover X 射线粉末衍射仪 (XRD) 分析样品的物相组成; 利用 Optima 7000 电感耦合 等离子体发射光谱仪分析浸渍液中残余 $\mathrm{Cu}^{2+}$ 浓度; 利用 Nicolet 5700 傅里叶变换红外光谱仪(FT-IR)分 析样品的重要官能团; 利用 Tristar3020 比表面分析 仪分析样品的比表面积和孔径分布; 利用 STA409PC 同步热分析仪(TGA)检测材料的受热变化过程; 利 用 ESCALAB 250XI X 射线光电子能谱(XPS)分析 样品表面的元素组成和价态; 利用 BRUKER S4 PIONEER X 射线荧光光谱仪 (XRF)测试固体样品的 化学组成。

\section{$1.4 \mathrm{Cu} @$ PAL 对 $\mathrm{I}^{-}$的吸附实验}

由于放射性碘化物的放射性和毒性, 本研究选 用化学性质完全相同的非放射性的 ${ }^{127} \mathrm{I}^{-}$。在吸附实 验中, 取 $50 \mathrm{mg}$ 吸附剂加入至 $50 \mathrm{~mL} \mathrm{NaI}$ 溶液中, 敞 口暴露于空气中, 在振荡器中以 $150 \mathrm{r} / \mathrm{min}$ 速度摇 动。吸附完成后, 离心分离, 上层清液经 $0.45 \mu \mathrm{m}$ 微 孔滤膜过滤后, 利用紫外分光光度法于 $227 \mathrm{~nm}$ 处测 试其碘离子浓度。多次水洗下层固体至无碘离子检 出后, 烘干、研磨成粉备用。

\section{2 结果与讨论}

\section{1 样品表征}

对 PAL、Cu ${ }^{2+} @$ PAL 和 $\mathrm{Cu} @$ PAL 样品进行形貌 表征。如图 1 所示, PAL 经过硝酸铜溶液浸渍和还原 后微观上仍呈长 $0.5 \sim 2.0 \mu \mathrm{m}$ 的高长径比棒状结构, 表明该制备方法未破坏 PAL 的原有结构。天然凹土 (图 1(a)) 含有少量石英和碳酸钻等细微块状颗粒, 而浸渍后(图 1(b)) 和还原后(图 1(c))样品中的块状颗 粒明显减少, XRF 测试结果(表 1)也显示还原后样品 的钙含量明显降低, 这是因为制备过程中的弱酸性 硝酸铜溶液可分解 PAL 中的碳酸钙杂质, 提高了 PAL 的纯度。Cu@PAL 样品的元素谱图(图 1(d))表 明, $\mathrm{Cu}$ 的掺杂量约为 $8.32 \mathrm{wt} \%$, 这与 ICP-AES 测得 的 $7.52 \mathrm{wt} \%$ 含量较为接近。

对比 PAL、Cu ${ }^{2+} @ \mathrm{PAL}$ 和 $\mathrm{Cu} @ \mathrm{PAL}$ 样品的 XRD 图谱(图 2(a))发现, PAL 结构未被破坏, 在 $\mathrm{Cu}^{2+} @ \mathrm{PAL}$ 样品中新增的 $2 \theta=12.8^{\circ} 、 25.8^{\circ} 、 33.5^{\circ}$ 和 $36.5^{\circ}$ 衍射 峰分别对应 $\mathrm{Cu}_{2}(\mathrm{OH})_{3}\left(\mathrm{NO}_{3}\right)$ (JCPDS 54-0747) 的 (001)、(002)、(120)和(121) 晶面, 这是因为 $\mathrm{Cu}^{2+}$ 取 代了 PAL 结构中的可交换阳离子 $\left(\mathrm{Na}^{+} 、 \mathrm{~K}^{+} 、 \mathrm{Ca}^{2+}\right.$ 、
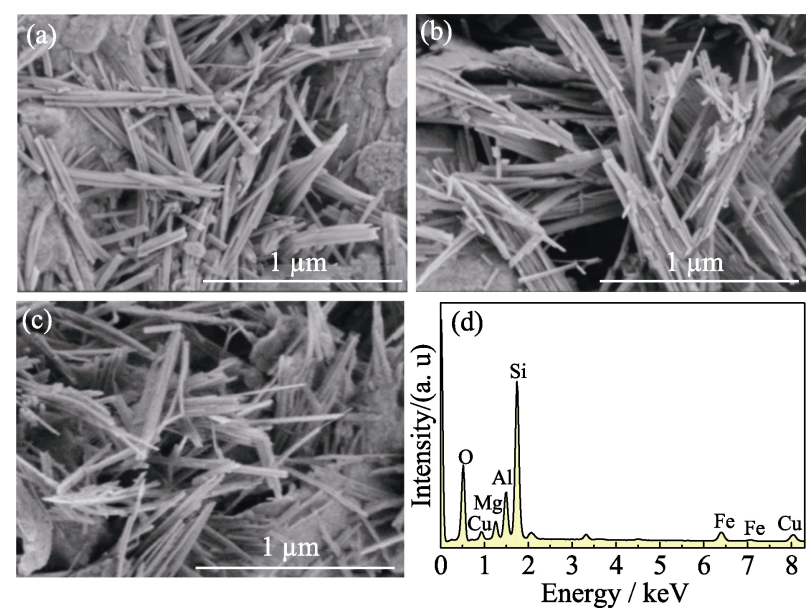

图 1 样品(a)PAL, (b)Cu $\mathrm{Cu}^{2+} @ \mathrm{PAL},(\mathrm{c}) \mathrm{Cu} @ \mathrm{PAL}$ 的 SEM 照片 及(d) $\mathrm{Cu} @$ PAL 的 EDX 谱图

Fig. 1 SEM images of the samples (a) PAL, (b) $\mathrm{Cu}^{2+} @ \mathrm{PAL}$, (c) $\mathrm{Cu} @$ PAL and (d) EDX pattern of $\mathrm{Cu} @$ PAL

表 1 PAL 和 Cu@PAL 的化学组成(XRF)/wt $\%$ Table1 Chemical analysis (XRF) of PAL and $\mathrm{Cu} @$ PAL $/$ wt \%

\begin{tabular}{crc}
\hline Composition & \multicolumn{1}{c}{ PAL } & $\mathrm{Cu} @$ PAL \\
\hline $\mathrm{SiO}_{2}$ & 60.96 & 59.87 \\
$\mathrm{MgO}$ & 9.36 & 8.12 \\
$\mathrm{Al}_{2} \mathrm{O}_{3}$ & 12.20 & 11.07 \\
$\mathrm{Fe}_{2} \mathrm{O}_{3}$ & 8.00 & 7.51 \\
$\mathrm{CaO}$ & 5.55 & 0.46 \\
$\mathrm{CuO}$ & 0.01 & 9.83 \\
$\mathrm{Na}_{2} \mathrm{O}$ & 0.12 & 0.01 \\
$\mathrm{LOI}$ & 3.80 & 3.13 \\
\hline
\end{tabular}
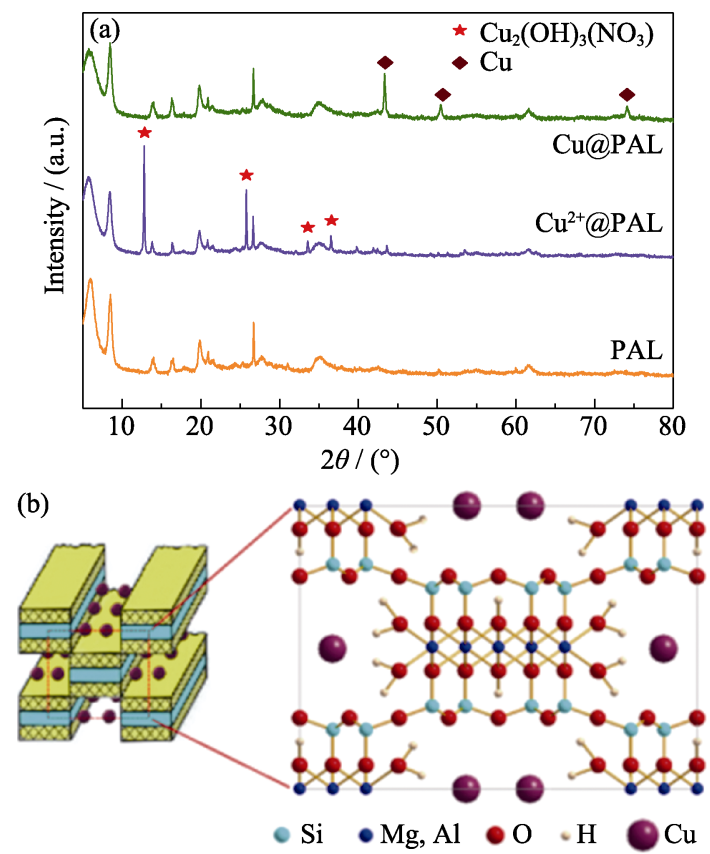

图 2 (a)样品的 XRD 图谱和(b)Cu@PAL 的结构示意图 Fig. 2 (a) XRD patterns of the samples and (b) structural schematic diagram of $\mathrm{Cu} @ \mathrm{PAL}$ 
$\mathrm{Mg}^{2+}$ 等)后与八面体层中间阳离子配位的结构水 $(\mathrm{OH})$ 结合造成的 ${ }^{[17-18]}$ 。经氢气还原后, $\mathrm{Cu}_{2}(\mathrm{OH})_{3}\left(\mathrm{NO}_{3}\right)$ 的特征峰消失, 在 $2 \theta=43.3^{\circ} 、 50.4^{\circ}$ 和 $74.1^{\circ}$ 处出现属 于单质 $\mathrm{Cu}$ 的(111)、(200)和(220)晶面(JCPDS 04-0836) 的新衍射峰。这表明在制备过程中, $\mathrm{Cu}^{2+}$ 先与 $\mathrm{PAL}$ 结构水结合, 随后经氢气还原转化为单质 $\mathrm{Cu}$, 并负 载于 PAL 结构中(图 2(b))。

$\mathrm{Cu}^{2+} @$ PAL 样品的 TEM 照片和元素分布图如 图 3 所示, PAL 经浸渍后仍保持棒状结构, 各元素均 匀分布于 PAL 晶体结构中。图 4 是 $\mathrm{Cu} @ \mathrm{PAL}$ 样品 的 TEM 照片、元素分布以及 HRTEM 照片。图 4(a,g) 的 TEM 照片表明，在 PAL 的棒状晶束中负载有纳 米颗粒。图 4(b f)的元素分布图表明, 经氢气还原 后，各元素仍均匀分布于 PAL 晶体结构中。HRTEM 照片(图 4(h))显示颗粒状物晶格间距为 $0.2088 \mathrm{~nm}$, 对应单质铜的(111)晶面 ${ }^{[19]}$, 再次表明纳米铜颗粒均 匀负载于 PAL 结构中。

图 5 是 $\mathrm{Cu} @ \mathrm{PAL}$ 的 XPS 谱图, 结合能由污染碳 $\mathrm{C} 1 \mathrm{~s}(284.8 \mathrm{eV})$ 校准。对 $\mathrm{Cu} 2 \mathrm{p}$ 能谱进行高分辨扫描,
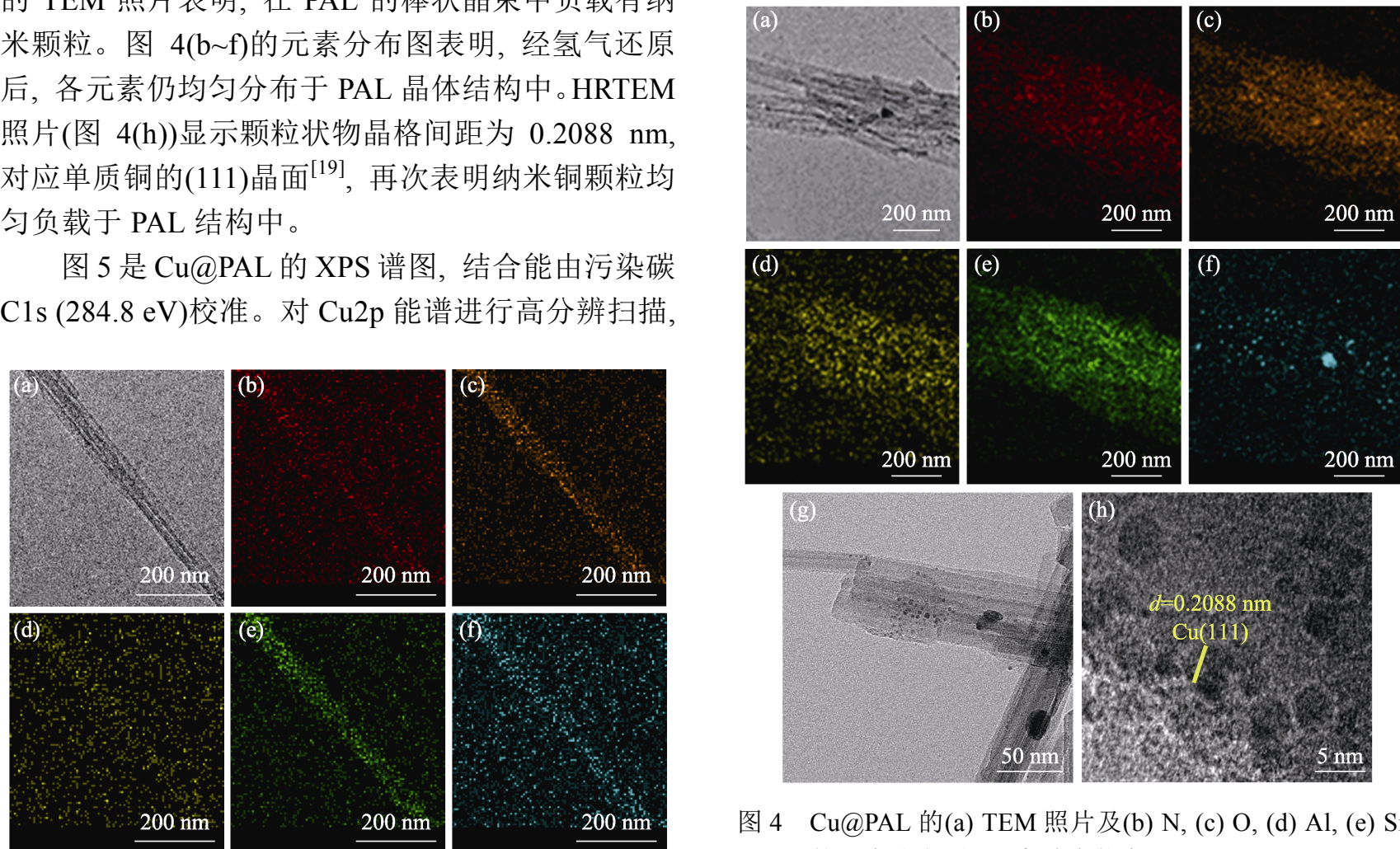

图 $4 \mathrm{Cu} @$ PAL 的(a) TEM 照片及(b) N, (c) O, (d) Al, (e) Si, (f) $\mathrm{Cu}$ 的元素分布图, 更高放大倍率 TEM (g) 和 HRTEM (h) 照片

Fig. 4 (a) TEM image of $\mathrm{Cu} @ \mathrm{PAL}$ and elemental mappings of (b) N, (c) $\mathrm{O}$, (d) $\mathrm{Al}$, (e) $\mathrm{Si}$, and (f) $\mathrm{Cu}$; and high magnification TEM $(\mathrm{g})$ and HRTEM $(\mathrm{h})$ images of $\mathrm{Cu} @$ PAL

图 $3 \mathrm{Cu}^{2+} @$ PAL 的(a) TEM 照片及(b) N, (c) O, (d) Al, (e) Si, (f) $\mathrm{Cu}$ 的元素分布图

Fig. 3 (a) TEM image of $\mathrm{Cu}^{2+} @$ PAL and elemental mappings of (b) N, (c) O, (d) Al, (e) Si, and (f) $\mathrm{Cu}$

如图 5(b)所示, 952.2 和 $932.3 \mathrm{eV}$ 两处峰对应铜单质 的 $\mathrm{Cu} 2 \mathrm{p} 1 / 2$ 和 $\mathrm{Cu} 2 \mathrm{p} 3 / 2$ 特征峰, 954.2 和 $934.5 \mathrm{eV}$ 处 峰分别归属于亚铜的 $\mathrm{Cu} 2 \mathrm{p} 1 / 2$ 和 $\mathrm{Cu} 2 \mathrm{p} 3 / 2^{[12]}$, 表明 $\mathrm{Cu} @ \mathrm{PAL}$ 表面的铜主要以铜单质存在并伴随少量亚 铜, 与其它铜基材料 ${ }^{[12,20]}$ 相比，由于 $\mathrm{Cu}$ 负载于 $\mathrm{PAL}$

图 6(a)是样品的热失重曲线。低于 $140{ }^{\circ} \mathrm{C}$ 的失 重是由材料表面物理吸附水的脱除引起的 ${ }^{[21]}$, 这是 PAL 和 $\mathrm{Cu} @ \mathrm{PAL}$ 都具有的失重阶段, 但 $\mathrm{Cu} @ \mathrm{PAL}$ 比 PAL 少失重约 5.7\%; 在孔道中沸石水和第一结晶 水的失重阶段 ${ }^{[22]}\left(140 \sim 374{ }^{\circ} \mathrm{C}\right.$ 范围), $\mathrm{Cu} @ \mathrm{PAL}$ 基本
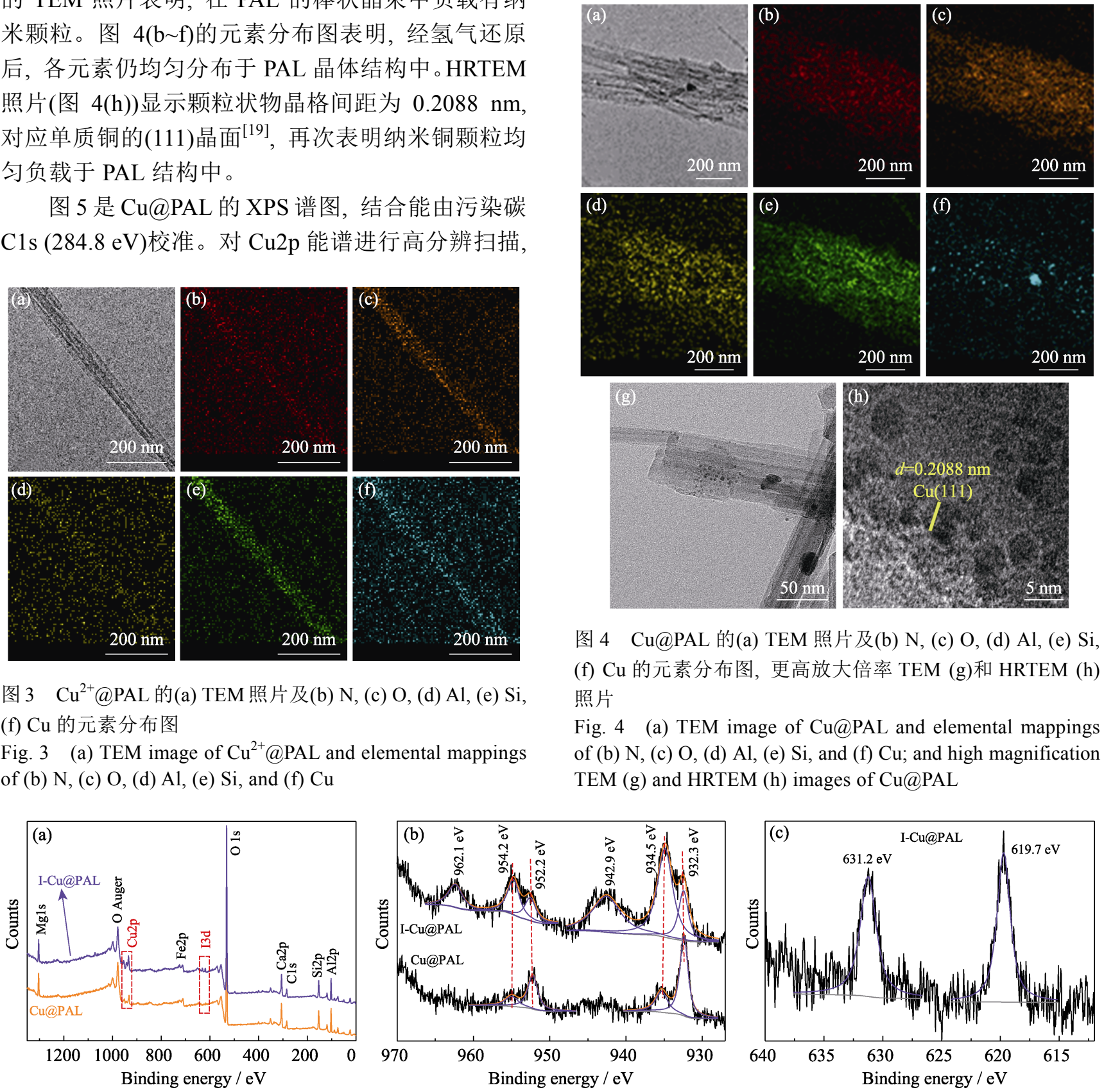

图 5 样品的 XPS 谱图

Fig. 5 XPS spectra of the samples

(a) Survey scans; (b) Cu2p core level spectra; (c) I 3d core level spectrum 

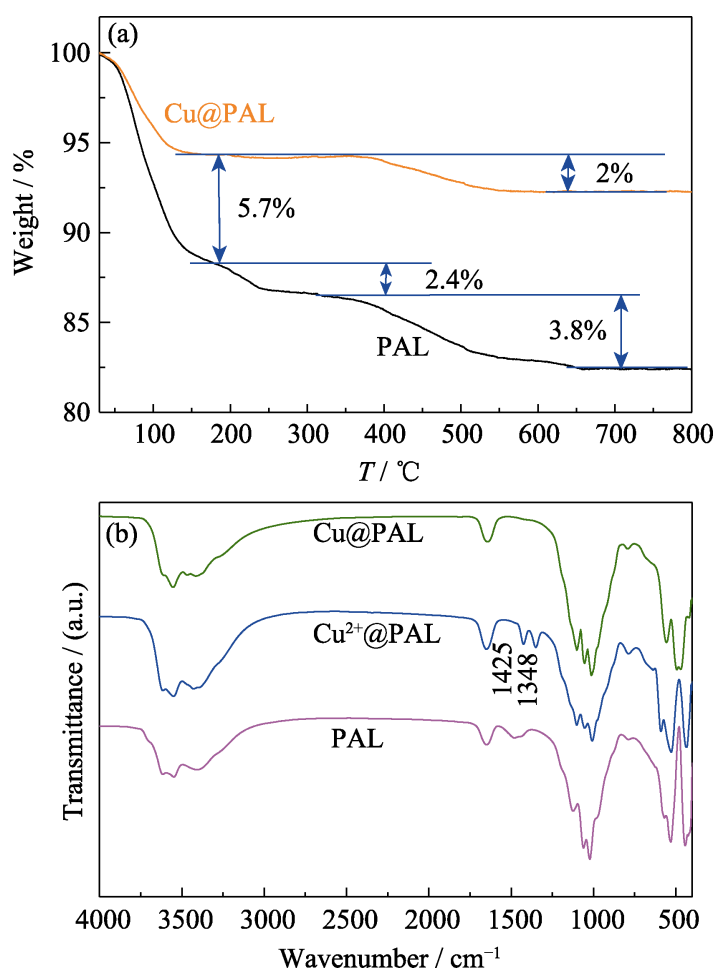

图 6 样品的(a)热失重曲线和(b) FT-IR 谱图

Fig. 6 (a) TGA curves and (b) FT-IR spectra of the samples

无重量损失，而 PAL 失重了 $2.4 \%$ 左右。两阶段的失 重和刚好与 $\mathrm{Cu} @ \mathrm{PAL}$ 中铜掺杂量 $(7.5 \mathrm{wt} \%)$ 相吻合。 此外，在第二结晶水和层间结构水的第三失重阶 段 ${ }^{[23]}$ (374 650 ${ }^{\circ} \mathrm{C}$ 范围), $\mathrm{Cu} @$, PAL 失重量也明显下 降, 再次表明 $\mathrm{Cu}$ 与层间结构水的羟基结合, 并占据 了 PAL 孔道结构中属于沸石水和结构水的位置。

样品的 FT-IR 谱图(图 6(b))再次表明改性前后 的 PAL 特征峰未发生变化。 $\mathrm{Cu}^{2+} @ \mathrm{PAL}$ 的红外光谱 在 1425、1348 $\mathrm{cm}^{-1}$ 处出现的吸收峰是 $\mathrm{Cu}_{2}(\mathrm{OH})_{3}\left(\mathrm{NO}_{3}\right)$ 中 $\mathrm{NO}_{3}{ }^{-}$的对称伸缩振动和反对称伸缩振动吸收峰 ${ }^{[24]}$ 。 同时位于 $1440 \mathrm{~cm}^{-1}$ 处的碳酸钙杂质的 $\mathrm{CO}_{3}{ }^{2-}$ 吸收 峰 ${ }^{[25]}$ 掺杂后消失, 与 SEM、XRF 结论相一致。

图 7 是 PAL 和 $\mathrm{Cu} @$ PAL 样品的 $\mathrm{N}_{2}$ 吸附脱附等 温和孔径分布曲线。如图 7(a)所示, 根据 IUPAC 的 分类 ${ }^{[26]}, \mathrm{PAL}$ 和 $\mathrm{Cu} @ \mathrm{PAL}$ 的吸附脱附等温线属于IV 型等温线, 滞留环为 $\mathrm{H} 3$ 型, 表明 PAL 和 $\mathrm{Cu} @ \mathrm{PAL}$ 中存在介孔。PAL 和 $\mathrm{Cu} @ \mathrm{PAL}$ 的比表面积分别为 117.5 和 $113.4 \mathrm{~m}^{2} / \mathrm{g}$ 。PAL 和 $\mathrm{Cu} @$ PAL 的孔径(图 7(b)) 主要分布于 2 4 和 20 40 nm 的介孔范围内, 平均孔 径分别为 15.7 和 $15.0 \mathrm{~nm}$, 表明铜负载于 PAL 结构 中使比表面和孔径稍微下降。

\section{$2.2 \mathrm{pH}$ 对吸附性能的影响}

实验研究了 $\mathrm{pH}$ 对 $\mathrm{Cu} @ \mathrm{PAL}$ 的吸附性能影响, $\mathrm{I}^{-}$ 初始浓度设置为 $2 \mathrm{mmol} / \mathrm{L}$ 。结果如图 8(a)所示, 由 于 PAL 自身带有负电荷, 因而对碘离子基本无吸附
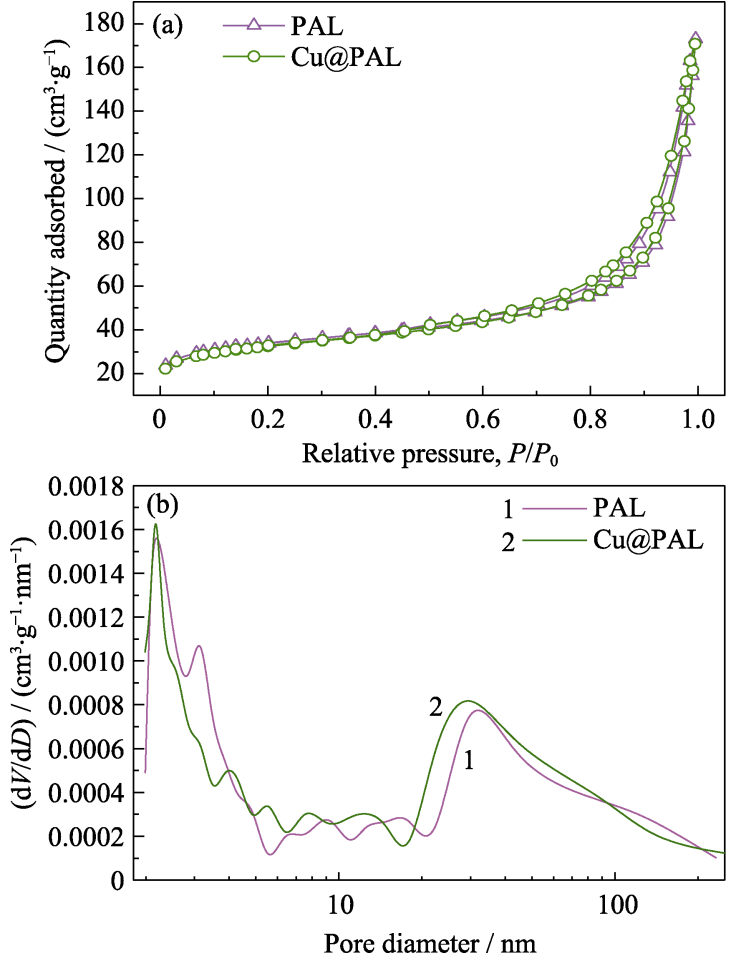

图 7 样品的(a) $\mathrm{N}_{2}$ 吸附脱附等温线和(b)孔径分布

Fig. 7 (a) $\mathrm{N}_{2}$ adsorption-desorption isotherms and (b) pore diameter distributions of the samples
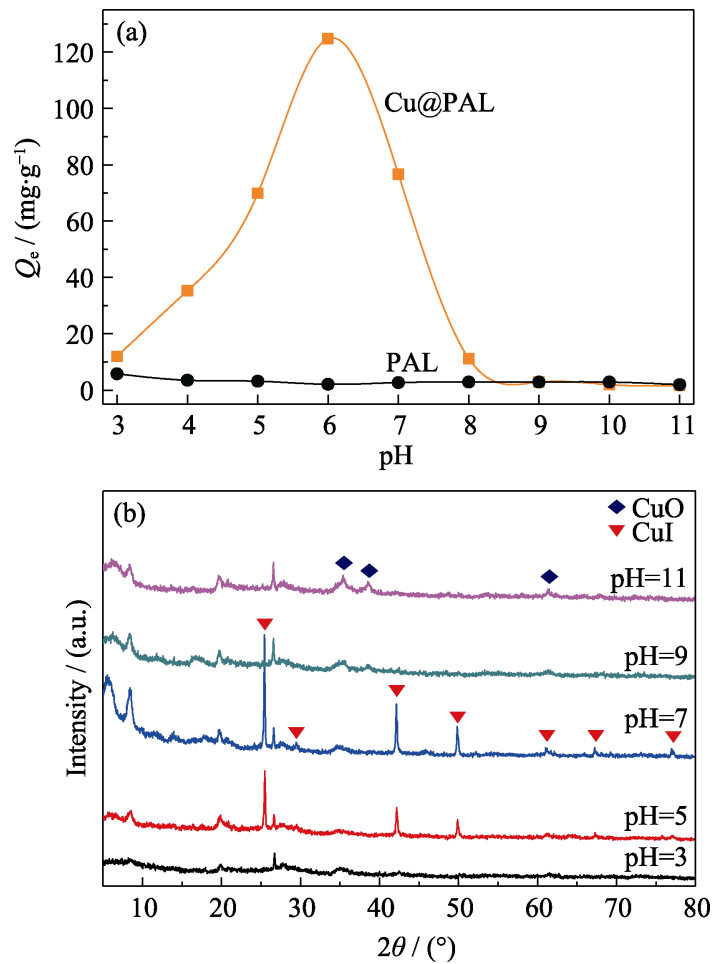

图 8 (a)pH 对 $\mathrm{Cu} @$ PAL 吸附性能的影响和(b)产物的 XRD 谱图 Fig. 8 (a) Adsorption capacity of $\mathrm{I}^{-}$anions on $\mathrm{Cu} @ \mathrm{PAL}$ and (b) XRD patterns of adsorption products in the solution with different $\mathrm{pH}$

性能。当 $\mathrm{pH}=6$ 时, $\mathrm{Cu} @ \mathrm{PAL}$ 对 I的吸附容量最大, 可达 $124.8 \mathrm{mg} / \mathrm{g}$; 当增加或降低 $\mathrm{pH}$ 时，吸附容量逐 渐下降。当 $\mathrm{pH}>8$ 时, $\mathrm{Cu} @ \mathrm{PAL}$ 基本无吸附性能。 
利用 XRD 分析了 $\mathrm{Cu} @ \mathrm{PAL}$ 吸附后产物(图 8(b))。 在中性和弱酸性条件下, $\mathrm{Cu} @ \mathrm{PAL}$ 吸附 $\mathrm{I}^{-}$后, 单质 $\mathrm{Cu}$ 的特征峰消失, 在 $2 \theta=25.5^{\circ} 、 29.5^{\circ} 、 42.2^{\circ} 、 49.9^{\circ}$ 、 $61.2^{\circ} 、 67.4^{\circ} 、 77.2^{\circ}$ 处出现 $\mathrm{CuI}(\mathrm{JCPDS}$ 06-0246)的 (111)、(200)、(220)、(311)、(400)、(331)、(422)晶 面的衍射峰, 表明吸附后的 $\mathrm{I}$ 以 $\mathrm{CuI}$ 形式赋存于产 物中。由于强酸性可将 PAL 分解为无定形二氧化硅, 因而随着酸性增加, PAL 特征峰逐渐变弱直至消失, 样品吸附性能也随之降低, 说明吸附过程中 PAL 的 载体作用也至关重要。碱性条件下，在 $2 \theta=35.5^{\circ}$ 、 38. $7^{\circ}$ 和 61.5\%处出现 CuO(JCPDS 45-0937)的(111)、 (111)和(113)晶面的衍射峰, 表明碱性条件可将单 质 $\mathrm{Cu}$ 氧化成 $\mathrm{CuO}$, 从而阻止了复合材料对碘离子 的有效吸附。因此, $\mathrm{Cu} @ \mathrm{PAL}$ 可应用于中性或弱酸 性环境的 I吸附。

\subsection{Cu@PAL 的等温吸附性能}

$\mathrm{Cu} @ \mathrm{PAL}$ 在 $\mathrm{pH}=7$ 条件下的等温吸附性能如 图 9(a)所示。PAL 对 $\mathrm{I}^{-}$基本无吸附效果。当溶液中 $\mathrm{I}^{-}$的起始浓度从 $0.4 \mathrm{mmol} / \mathrm{L}$ 增大至 $12 \mathrm{mmol} / \mathrm{L}$, $\mathrm{Cu} @ \mathrm{PAL}$ 对 $\mathrm{I}^{-}$的吸附容量也从 $13.7 \mathrm{mg} / \mathrm{g}$ 增加到 $116.1 \mathrm{mg} / \mathrm{g}$ 左右。与此前报道的铜基碘离子吸附剂 相比(表 2), $\mathrm{Cu} @ \mathrm{PAL}$ 的吸附容量和 $\mathrm{Cu}$ 利用率均为 最佳。

采用两种经典等温吸附模型 Langmuir 和 Freundlich 模型进一步评估 $\mathrm{Cu} @ \mathrm{PAL}$ 的吸附过程:

Langmuir 等温方程: $\frac{C_{\mathrm{e}}}{Q_{\mathrm{e}}}=\frac{1}{K_{1} Q_{\mathrm{m}}}+\frac{C_{\mathrm{e}}}{Q_{\mathrm{m}}}$

Freundlich 等温方程: $\ln Q_{\mathrm{e}}=\frac{1}{n} \ln C_{\mathrm{e}}+\ln K_{\mathrm{f}}$

式中 $Q_{\mathrm{e}}$ 为平衡吸附容量, 单位为 $\mathrm{mg} / \mathrm{g} ; Q_{\mathrm{m}}$ 为理论最 大吸附容量, 单位为 $\mathrm{mg} / \mathrm{g} ; C_{\mathrm{e}}$ 为平衡浓度, 单位为 $\mathrm{mmol} / \mathrm{L} ; K_{1}$ 和 $K_{\mathrm{f}}$ 分别为 Langmuir 平衡常数和 Freundlich 平衡常数, $n$ 为 Freundlich 线性指数。

两种吸附模型的拟合结果如图 9(b, c) 和表 3 所
示, 对比 Freundlich 模型和 Langmuir 模型拟合的 $R^{2}$, 说明 Langmuir 模型更适于描述 $\mathrm{Cu} @ \mathrm{PAL}$ 对 $\mathrm{I}^{-}$的吸 附, $\mathrm{Cu} @ \mathrm{PAL}$ 对 $\mathrm{I}^{-}$的吸附为单分子层的化学吸附。由 Langmuir 模型拟合得到 $\mathrm{Cu} @ \mathrm{PAL}$ 对 I 的理论最大吸 附量为 $1.03 \mathrm{mmol} / \mathrm{g}(130.7 \mathrm{mg} / \mathrm{g})$, 与实验所得的 $116.1 \mathrm{mg} / \mathrm{g}$ 吸附量基本吻合。

\section{$2.4 \mathrm{Cu} @$ PAL 的抗氧化性能测试}

为评估 $\mathrm{Cu} @ \mathrm{PAL}$ 的抗氧化性能，在室温下分别 将 $\mathrm{Cu} @ \mathrm{PAL}$ 和纳米铜粉暴露于空气中一定时间后

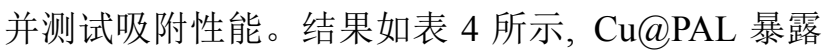
于空气 $6 \mathrm{~d}$ 后吸附容量仅下降 $20.9 \%$, 而纳米铜下降 了 $62.3 \%$, 显示 $\mathrm{Cu} @ \mathrm{PAL}$ 具有较强的抗氧化性能。 铜表面易被针化为 $\mathrm{CuO}$, 从而导致铜基吸附剂吸附 率下降 ${ }^{[29]}$ 。而 $\mathrm{Cu} @ \mathrm{PAL}$ 中铜虽为纳米颗粒, 但其负 载于 PAL 的层间, 减少了与空气接触的几率, 因而 具有较好的抗氧化性能。

\section{5 吸附机理}

为探究 $\mathrm{Cu} @ \mathrm{PAL}$ 对碘离子的吸附机理, 对吸附 后产物进行了表征测试。由图 8(b) XRD 图谱可知, 吸附后产物中含有较强的 $\mathrm{CuI}$ 特征峰。此外, I-Cu@PAL XPS 的 $\mathrm{Cu} 2 \mathrm{p}$ 高分辨扫描结果(图 5(b)) 显示, 属于单质铜的 952.2 和 $932.3 \mathrm{eV}$ 两处特征峰 减弱, 而归属于亚铜的 954.2 和 $934.5 \mathrm{eV}$ 处特征峰 增强, 并且新增位于 942.9 和 $962.1 \mathrm{eV}$ 处的两个卫 星峰属于 $\mathrm{CuO}^{[30]}$ 。表明吸附后中的铜主要以 $\mathrm{CuI}$ 形 式存在, 同时含有少量 $\mathrm{CuO} 。 \mathrm{I} 3 \mathrm{~d}$ 的高分辨扫描结果 (图 5(c))显示, 吸附后产物中碘仍是 $\mathrm{I}^{-}$。

图 10 是吸附剂 $\mathrm{Cu} @$ PAL 吸附碘离子后产物的 SEM 和 TEM 照片。对比吸附前后的形貌(图 1(c)和 图 10(a)) 可知吸附过程未破坏 PAL 的棒状结构。同 时, TEM 照片(图 10(b))显示吸附后的 CuI 产物仍均 匀分散于 PAL 的棒状结构中, HRTEM(图 10(c))照片 中 $0.3493 、 0.2139 \mathrm{~nm}$ 的晶格线分别属于 $\mathrm{CuI}$ 的(111) 和(220)晶面。因此, $\mathrm{Cu} @ \mathrm{PAL}$ 的吸附机理主要
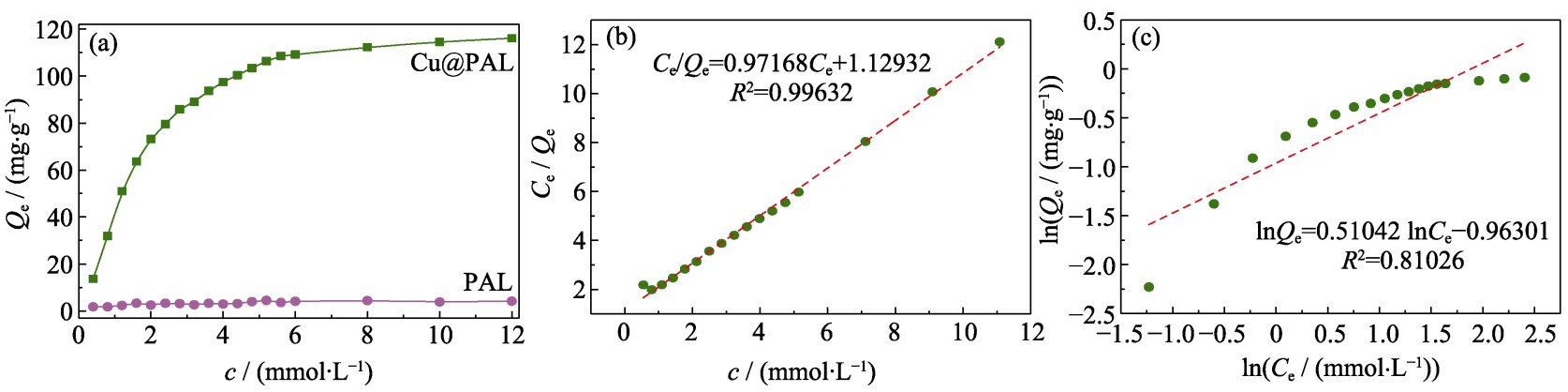

图 9 (a)样品的等温吸附曲线, $\mathrm{Cu} @ \mathrm{PAL}$ 等温吸附的(b) Langmuir 模型和(c) Frenudlich 模型模拟曲线

Fig. 9 (a) Adsorption isotherm of the samples, fitting curves of (b) Langmuir model and (c) Frenudlich model for adsorption isotherm of $\mathrm{Cu} @ \mathrm{PAL}$ 
表 2 铜基吸附剂碘离子吸附性能对比

Table 2 Comparison of several $\mathrm{Cu}$ based adsorbents for iodide adsorption

\begin{tabular}{lcccc}
\hline \multicolumn{1}{c}{ Adsorbent } & $\mathrm{pH}$ & $\begin{array}{c}Q_{\mathrm{e}} / \\
\left(\mathrm{mg} \cdot \mathrm{g}^{-1}\right)\end{array}$ & $\begin{array}{c}\text { Utilization } \\
\text { efficiency/\% }\end{array}$ & Ref. \\
\hline Cuprite sulfide & 7 & 6.1 & - & {$[27]$} \\
$\mathrm{Cu}{ }_{2} \mathrm{O} / \mathrm{Cu}-\mathrm{C}$ & 7 & 41.2 & 10.38 & {$[28]$} \\
$\mathrm{Hollow} \mathrm{Cu} / \mathrm{Cu}_{2} \mathrm{O}$ & 7 & 33.0 & 1.85 & {$[20]$} \\
Core-shell $\mathrm{Cu} / \mathrm{Cu}_{2} \mathrm{O}$ & 7 & 22.9 & 1.4 & {$[12]$} \\
$\mathrm{Cu}$ & 7 & 6.35 & 0.32 & {$[29]$} \\
$\mathrm{Cu} / \mathrm{PAL}$ & 7 & 116.1 & 71.98 & This work \\
\hline
\end{tabular}

表 $3 \mathrm{Cu} @$ PAL 等温吸附模型的拟合参数

Table 3 Isotherm parameters for the adsorption of $I^{-}$anions by $\mathrm{Cu} @$ PAL

\begin{tabular}{cccccccc}
\hline \multicolumn{3}{c}{ Langmuir model } & & \multicolumn{3}{c}{ Frenudlich model } \\
\cline { 1 - 3 } \cline { 5 - 7 }$Q_{\mathrm{m}} /\left(\mathrm{mg} \cdot \mathrm{g}^{-1}\right)$ & $K_{\mathrm{l}}$ & $R^{2}$ & & $K_{\mathrm{f}}$ & $1 / n$ & $R^{2}$ \\
\hline 1.02915 & 0.86041 & 0.99632 & & 0.38173 & 0.51042 & 0.81026 \\
\hline
\end{tabular}

为结构中纳米单质铜被溶液中溶解氧缓慢氧化成 $\mathrm{Cu}_{2} \mathrm{O}, \mathrm{Cu}_{2} \mathrm{O}$ 再与溶液中碘离子反应生成 $\mathrm{CuI}, \mathrm{PAL}$ 的孔道结构阻碍了铜的进一步氧化并为 $\mathrm{CuI}$ 产物提 供了附着载体。

\section{6 吸附动力学}

研究了初始浓度为 $2 \mathrm{mmol} / \mathrm{L}$ 的 $\mathrm{Cu} @ \mathrm{PAL}$ 的吸 附动力学性能。结果如图 11(a)所示, 吸附剂前 $2 \mathrm{~h}$
表 $4 \mathrm{Cu} @$ PAL 和纳米铜暴露于空气中的吸附性能

Table 4 Adsorption properties of Cu@PAL and nano $\mathrm{Cu}$ exposed to air

\begin{tabular}{rcc}
\hline \multirow{2}{*}{ Time/h } & \multicolumn{2}{c}{$Q_{\mathrm{e}} /\left(\mathrm{mg} \cdot \mathrm{g}^{-1}\right)$} \\
\cline { 2 - 3 } & $\mathrm{Cu} @$ PAL & Nano-Cu \\
\hline 0 & 74.2 & 234.7 \\
12 & 69.4 & 133.8 \\
24 & 68.1 & 110.4 \\
48 & 64.1 & 104.6 \\
72 & 60.2 & 96.3 \\
144 & 58.7 & 88.4 \\
\hline
\end{tabular}

的吸附速率较快, 随后变缓, 约 $8 \mathrm{~h}$ 后达到吸附平 衡。为进一步研究 $\mathrm{Cu} @$ PAL 吸附剂的吸附动力学行 为, 用伪一级动力学模型和伪二级动力学模型对吸 附实验数据进行了拟合分析：

$$
\begin{aligned}
& \text { 伪一级动力学模型: } \ln \left(Q_{\mathrm{m}}-Q_{t}\right)=\ln Q_{\mathrm{m}}-k_{1} t \\
& \text { 伪二级动力学模型: } \frac{t}{Q_{t}}=\frac{1}{k_{2} Q_{\mathrm{m}}^{2}}+\frac{1}{Q_{\mathrm{m}}} t
\end{aligned}
$$

式中 $t$ 为吸附时间, 单位为 $\mathrm{h} ; Q_{t}$ 为 $t$ 时刻的吸附容 量, 单位为 $\mathrm{mg} / \mathrm{g} ; k_{1}$ 和 $k_{2}$ 分别为伪一级速率常数和 伪二级速率常数, 单位为 $\mathrm{g} /\left(\mathrm{mmol} \cdot \mathrm{g}^{-1}\right)$ 。

两种动力学模型拟合结果如图 11(b, c) 和表 5 所 示, 对比伪一、二级动力学模型拟合 $\mathrm{Cu} @$ PAL 吸附 $\mathrm{I}^{-}$热力学过程的 $R^{2}$, 表明伪二级动力学模型更适于
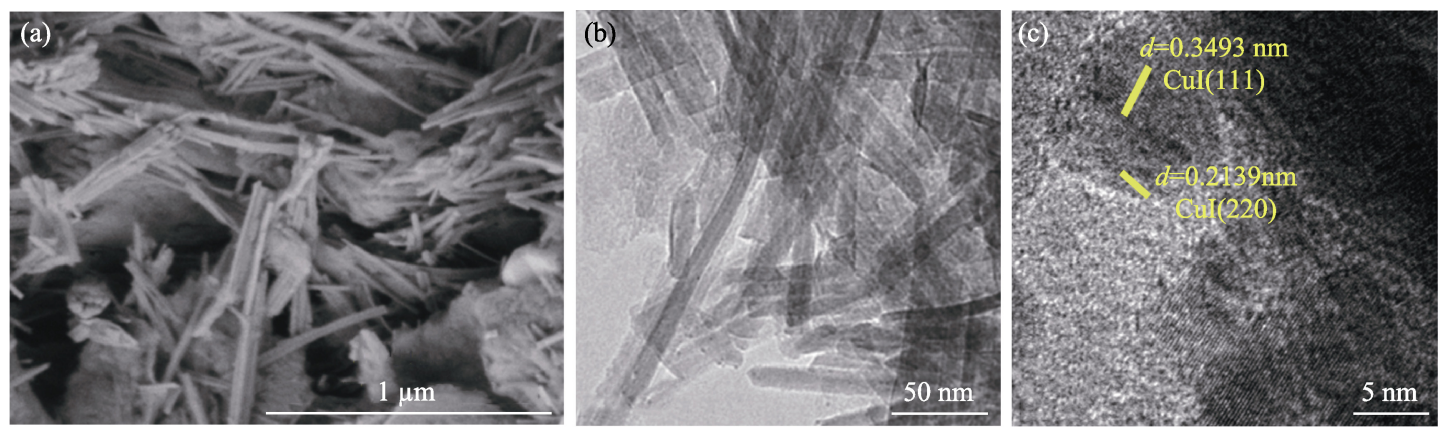

图 10 I-Cu@PAL 的 SEM(a), TEM(b) 和 HRTEM (c) 照片

Fig. 10 SEM (a) and TEM (b) and HRTEM (c) images of I-Cu@PAL
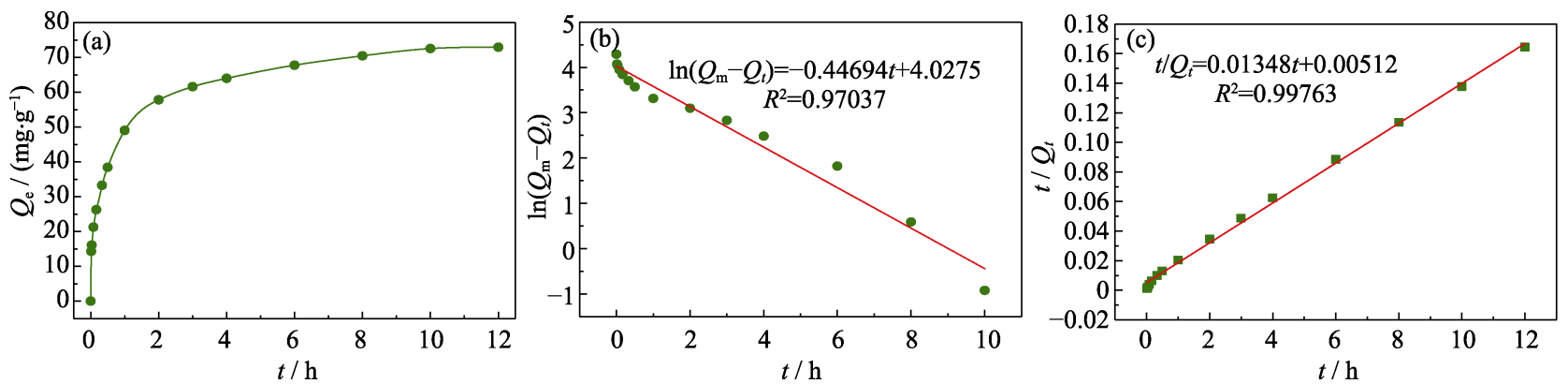

图 $11 \mathrm{Cu} @ \mathrm{PAL}$ 的吸附动力学曲线( $\mathrm{a}$ )以及吸附动力学曲线的伪一级动力学模型 (b) 和伪二级动力学模型 $(\mathrm{c})$

Fig. 11 Absorption kinetic curve (a), fitting curves of the pseudo first order kinetic model (b) and pseudo second order kinetic model (c) for adsorption kinetic of $\mathrm{Cu} @ \mathrm{PAL}$ 
表 $5 \mathrm{Cu} @$ PAL 的吸附动力学拟合参数

Table 5 Kinetic parameters for the adsorption of $\mathrm{I}^{-}$by $\mathrm{Cu} @$ PAL

\begin{tabular}{ccccccc}
\hline \multicolumn{3}{c}{ Pseudo-first-order } & & \multicolumn{3}{c}{ Pseudo-second-order } \\
\cline { 1 - 2 } \cline { 5 - 7 } $\begin{array}{c}Q_{\mathrm{m}} / \\
\left(\mathrm{mg} \cdot \mathrm{g}^{-1}\right)\end{array}$ & $\begin{array}{c}k_{1} /(\mathrm{g} \cdot(\mathrm{mmol} \cdot \\
\left.\left.\mathrm{g}^{-1}\right)^{-1}\right)\end{array}$ & $R^{2}$ & & $\begin{array}{c}Q_{\mathrm{m}} / \\
\left(\mathrm{mg} \cdot \mathrm{g}^{-1}\right)\end{array}$ & $\begin{array}{c}k_{2} /(\mathrm{g} \cdot(\mathrm{mmol} \\
\left.\left.\mathrm{g}^{-1}\right)^{-1}\right)\end{array}$ & $R^{2}$ \\
\hline 56.10 & 0.4469 & 0.9704 & 74.1840 & 0.03549 & 0.9976 \\
\hline
\end{tabular}

描述 $\mathrm{Cu} @ \mathrm{PAL}$ 对 $\Gamma$ 吸附的动力学过程, 表明 $\mathrm{Cu} @ \mathrm{PAL}$ 对 $\Gamma^{-}$的吸附过程更受化学吸附机理的控制。此外, 由伪二级动力学模型拟合得到 $\mathrm{Cu} @ \mathrm{PAL}$ 对初始浓 度为 $2 \mathrm{mmol} / \mathrm{L}$ 的 $\mathrm{I}^{-}$的最大吸附量为 $74.2 \mathrm{mg} / \mathrm{g}$, 这与 实验所测数据 $(72.99 \mathrm{mg} / \mathrm{g})$ 相近。

\section{7 干扰离子对吸附的影响}

研究了不同干扰离子对 $\mathrm{Cu} @ \mathrm{PAL}$ 吸附性能的 影响, Г初始浓度设置为 $1 \mathrm{mmol} / \mathrm{L}$, 干扰离子浓度分 别为 $10 \mathrm{mmol} / \mathrm{L}$ 。实验结果如图 12 所示, 当有 $\mathrm{SO}_{4}{ }^{2-}$ 、 $\mathrm{NO}_{3}{ }^{-}$和 $\mathrm{Cl}^{-}$干扰时, $\mathrm{Cu} @ \mathrm{PAL}$ 对 I 的吸附容量稍有降 低但幅度较小, 表明 $\mathrm{Cu} @ \mathrm{PAL}$ 吸附 $\mathrm{I}^{-}$时对 $\mathrm{SO}_{4}{ }^{2-}$ 、 $\mathrm{NO}_{3}{ }^{-}$和 $\mathrm{Cl}^{-}$具有较强的抗干扰能力。而当溶液存有 $\mathrm{CO}_{3}{ }^{2-}$ 时, $\mathrm{Cu} @ \mathrm{PAL}$ 对 $\mathrm{I}^{-}$的吸附容量快速下滑至 $2.9 \mathrm{mmol} / \mathrm{g}$, 这是因为溶液中的 $\mathrm{CO}_{3}{ }^{2-}$ 水解形成碱性 环境, 不利于 $\mathrm{Cu} @ \mathrm{PAL}$ 对 $\mathrm{I}^{-}$的吸附, 与 $\mathrm{pH}$ 对吸附影 响的研究结果相吻合。 $\mathrm{Na}^{+} 、 \mathrm{~K}^{+}$对吸附性能几乎无影 响, 而 $\mathrm{Mg}^{+} 、 \mathrm{Zn}^{2+} 、 \mathrm{Al}^{3+}$ 可显著提升吸附剂的吸附性 能, 这是因为 $\mathrm{Mg}^{+} 、 \mathrm{Zn}^{2+} 、 \mathrm{Al}^{3+}$ 易水解形成酸性环境。
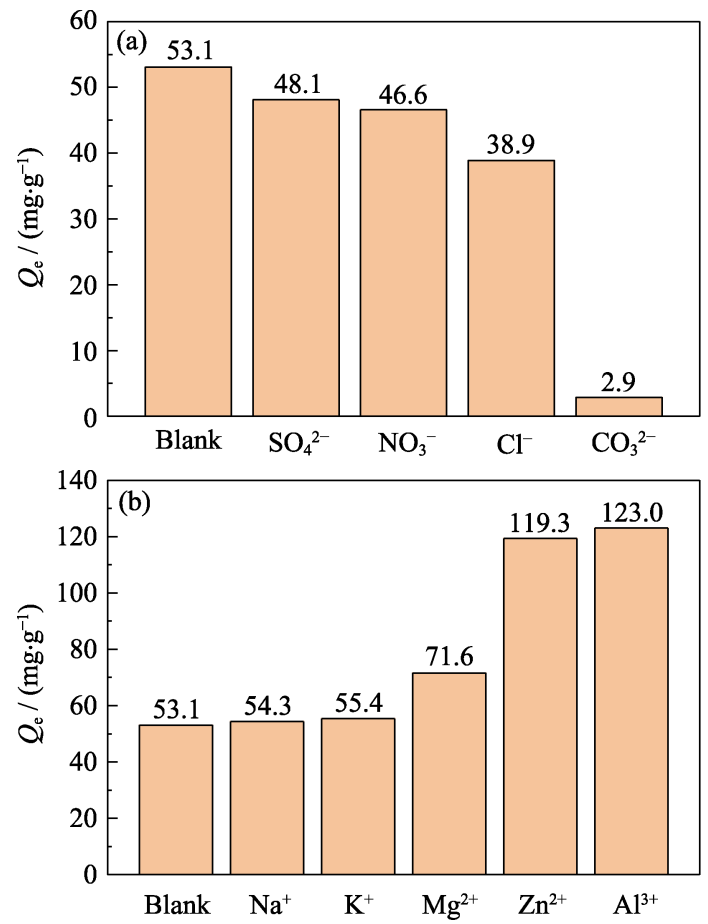

图 12 (a)阴离子和(b)阳离子对 $\mathrm{Cu} @$ PAL 吸附性能的影响 Fig. 12 Effect of (a) anion and (b) cation on the adsorption of $\mathrm{Cu} @$ PAL
表 $6 \mathrm{Cu} @$ PAL 和纳米 $\mathrm{Cu}$ 吸附后产物的脱附解析率

Table 6 Leaching or desorption efficiencies of $\mathrm{Cu} @ P A L$ and nano-Cu after adsorption

\begin{tabular}{ccc}
\hline Adsorbent & $c_{\mathrm{NaCl}}\left(\mathrm{mol} \cdot \mathrm{L}^{-1}\right)$ & Desorption efficiency $/ \%$ \\
\hline \multirow{2}{*}{$\mathrm{Nu} @ \mathrm{PAL}$} & 0 & 21.3 \\
& 0.1 & 32.1 \\
\hline \multirow{2}{*}{ Nano-Cu } & 0 & 87.4 \\
& 0.1 & 94.1 \\
\hline
\end{tabular}

\subsection{I-Cu@PAL 的脱附解析性能}

吸附后放射性产物的脱附易造成二次污染，而 $\mathrm{CuI}$ 光照不稳定, 因此研究了 $\mathrm{Cu} @ \mathrm{PAL}$ 和纳米 $\mathrm{Cu}$ 吸附后产物在纯水和 $\mathrm{NaCl}$ 干扰下的脱附解析性能。 实验结果如表 6 所示, 经光照 $12 \mathrm{~h}$ 后, I-Cu@PAL 在 纯水中 $\mathrm{I}^{-}$脱附率仅为 $21.3 \%$ 左右，而 $\mathrm{I}-\mathrm{Cu}$ 高达 $87.4 \%$; 在 $\mathrm{NaCl}$ 干扰条件下, I-Cu@PAL 的脱附率约 为 $32.1 \%$, 而 $\mathrm{I}-\mathrm{Cu}$ 高达 $94.1 \%$ 。这是由于 $\mathrm{Cu} @ \mathrm{PAL}$ 中的 $\mathrm{Cu}$ 赋存于 PAL 结构中, 因而吸附后产物可牢 固嵌入材料中, 从而降低了其被光照的几率。

\section{3 结论}

1)利用 PAL 结构中拥有可交换阳离子特性，成 功将铜牢固负载于 PAL 孔道结构中，显著提升了材 料在 I-吸附中的铜利用率和吸附容量, 并有效降低 了吸附产物的脱附率;

2)室温下, $\mathrm{Cu} @ \mathrm{PAL}$ 对 $\Gamma$ 的吸附平衡约为 $8 \mathrm{~h}$, 吸附容量最大可达 $116.1 \mathrm{mg} / \mathrm{g}$, 并具有较强的抗氧 化以及吸附后产物抗光照的性能;

3) $\mathrm{Cu} @ \mathrm{PAL}$ 宜在中性或弱酸性环境中对 I-吸附, 抗 $\mathrm{SO}_{4}{ }^{2-} 、 \mathrm{NO}_{3}{ }^{-}$和 $\mathrm{Cl}^{-}$干扰能力较强, $\mathrm{Mg}^{+} 、 \mathrm{Zn}^{2+} 、 \mathrm{Al}^{3+}$ 可提升吸附剂的吸附性能;

4) $\mathrm{Cu} @ \mathrm{PAL}$ 结构中纳米单质铜被溶解氧缓慢 氧化成与 $\mathrm{I}^{-}$反应的 $\mathrm{Cu}_{2} \mathrm{O}, \mathrm{PAL}$ 的孔道结构阻碍了铜 的进一步氧化并为 $\mathrm{CuI}$ 产物提供了附着载体, 从而 提升了材料的稳定性能。

\section{参考文献:}

[1] KBERGER T. Progress of renewable electricity replacing fossil fuels. Global Energy Interconnection, 2018, 1(1): 48-52.

[2] TRUESDALE V W, NAUSCH G, BAKER A. The distribution of iodine in the Baltic Sea during summer. Mar. Chem., 2001, 74(2): 87-98.

[3] GMEZ-GUZM N J M, HOLM E, NIAGOLOVA N, et al. Influence of releases of ${ }^{129} \mathrm{I}$ and ${ }^{137} \mathrm{Cs}$ from European reprocessing facilities in Fucus vesiculosus and seawater from the Kattegat and Skagerrak areas. Chemosphere, 2014, 108: 76-84.

[4] LI C, WEI Y, WANG X, et al. Efficient and rapid adsorption of 
iodide ion from aqueous solution by porous silica spheres loaded with calcined Mg-Al layered double hydroxide. Journal of the Taiwan Institute of Chemical Engineers, 2018, 85: 193-200.

[5] HOSKINS J S, KARANFIL T, SERKIZ S M. Removal and sequestration of iodide using silver-impregnated activated carbon. Environ. Sci. Technol., 2002, 36(4): 784-789.

[6] THEISS F L, AYOKO G A, FROST R L. Iodide removal using LDH technology. Chemical Engineering Journal, 2016, 296: 300-309.

[7] INOUE H. Effects of Co-ions on transport of iodide ions through a non-conventional anion exchange paper membrane. J. Membr. Sci., 2004, 228(2): 209-215.

[8] LIU S, WANG N, ZHANG Y, et al. Efficient removal of radioactive iodide ions from water by three-dimensional $\mathrm{Ag}_{2} \mathrm{O}-\mathrm{Ag} / \mathrm{TiO}_{2}$ composites under visible light irradiation. J. Hazard Mater, 2015, 284: 171-181.

[9] MAO P, LIU Y, JIAO Y, et al. Enhanced uptake of iodide on $\mathrm{Ag} @ \mathrm{Cu}_{2} \mathrm{O}$ nanoparticles. Chemosphere, 2016, 164: 396-403.

[10] CHOI M H, JEONG S W, SHIM H E, et al. Efficient bioremediation of radioactive iodine using biogenic gold nanomaterial-containing radiation-resistant bacterium, Deinococcus radiodurans R1. Chemical Communications, 2017, 53(28): 3937-3940.

[11] ZHANG W, LI Q, MAO Q, et al. Cross-linked chitosan microspheres: an efficient and eco-friendly adsorbent for iodide removal from waste water. Carbohydr. Polym., 2019, 209: 215-222.

[12] MAO P, QI L, LIU X, et al. Synthesis of $\mathrm{Cu} / \mathrm{Cu}_{2} \mathrm{O}$ hydrides for enhanced removal of iodide from water. J. Hazard Mater, 2017, 328: $21-28$.

[13] LIU L, LIU W, ZHAO X, et al. Selective capture of iodide from solutions by microrosette-like $\delta-\mathrm{Bi}_{2} \mathrm{O}_{3}$. ACS Applied Materials \& Interfaces, 2014, 6(18): 16082-16090.

[14] MAILEN J C, HORNER D E. Removal of iodine from reactor fuel solutions as insoluble $\mathrm{PdI}_{2}$. Nucl. Technol., 1977, 33(3): 260-263.

[15] BALSLEY S D, BRADY P V, KRUMHANSL J L, et al. Iodide retention by metal sulfide surfaces: cinnabar and chalcocite. Environ. Sci. Technol., 1996, 30(10): 3025-3027.

[16] YAQUAN W, FENG Y, JIANG J, et al. Designing of recyclable attapulgite for wastewater treatments: a review. ACS Sustainable Chemistry \& Engineering, 2018, 7(2): 1855-1869.

[17] CHEN H, ZHAO Y, WANG A. Removal of $\mathrm{Cu}(\mathrm{II})$ from aqueous solution by adsorption onto acid-activated palygorskite. J. Hazard
Mater, 2007, 149(2): 346-354.

[18] 王爱勤, 王文波, 郑易安, et al. 凹凸棒石棒晶束解离及其纳米 化功能复合材料. 北京: 科学出版社, 2014.

[19] ZHOU Y, HE J, WANG H, et al. Carbon nanofiber yarns fabricated from co-electrospun nanofibers. Materials \& Design, 2016, 95: 591-598.

[20] MAO P, JIANG J, PAN Y, et al. Enhanced uptake of iodide from solutions by hollow Cu-based adsorbents. Materials, 2018, 11(5): 769.

[21] MAO P, YU X, LIU K, et al. Rapid and reversible adsorption of radioactive iodide from wastewaters by green and low-cost palygorskite-based microspheres. J. Radioanal Nucl. Chem., 2020, 325(1): 303-313.

[22] ZHANG Y, YU C, HU P, et al. Mechanical and thermal properties of palygorskite poly(butylene succinate) nanocomposite. Applied Clay Science, 2016, 119: 96-102.

[23] CARAZO E, BORREGO-S NCHEZ A, GARC A-VILL N F, et al. Adsorption and characterization of palygorskite-isoniazid nanohybrids. Applied Clay Science, 2018, 160: 180-185.

[24] BA N, ZHU L, LI H, et al. 3D rod-like copper oxide with nanowire hierarchical structure: ultrasound assisted synthesis from $\mathrm{Cu}_{2}(\mathrm{OH})_{3} \mathrm{NO}_{3}$ precursor, optical properties and formation mechanism. Solid State Sciences, 2016, 53: 23-29.

[25] YAO S, ZHANG H, CHEN Z, et al. Promotion of graphitic carbon oxidation via stimulating $\mathrm{CO}_{2}$ desorption by calcium carbonate. $J$. Hazard Mater, 2019, 363: 10-15.

[26] THOMMES M, KANEKO K, NEIMARK A V, et al. Physisorption of gases, with special reference to the evaluation of surface area and pore size distribution (IUPAC Technical Report). Pure Appl. Chem., 2015, 87(9/10): 1051-1069.

[27] LEF VRE G, BESSI RE J, EHRHARDT J J, et al. Immobilization of iodide on copper(I) sulfide minerals. Journal of Environmental Radioactivity, 2003, 70(1/2): 73-83.

[28] ZHANG X, GU P, LI X, et al. Efficient adsorption of radioactive iodide ion from simulated wastewater by nano $\mathrm{Cu}_{2} \mathrm{O} / \mathrm{Cu}$ modified activated carbon. Chemical Engineering Journal, 2017, 322: 129-139.

[29] HAQ Z, BANCROFT G M, FYFE W S, et al. Sorption of iodide on copper. Environ. Sci. Technol., 1980, 14(9): 1106-1110.

[30] WU C K, YIN M, O'BRIEN S, et al. Quantitative analysis of copper oxide nanoparticle composition and structure by X-ray photoelectron spectroscopy. Chemistry of Materials, 2006, 18(25): 6054-6058. 